\section{The DDR is in}

\section{By Joanne Kotz, Senior Editor}

The clinical success of Pfizer Inc.'s crizotinib anaplastic lymphoma kinase (ALK) inhibitor illustrates that attacking oncogenic drivers can substantially improve outcomes in small groups of non-small cell lung cancer patients. Now, an international team has identified a similar target in squamous cell lung cancer-mutations in the tyrosine kinase DDR2 that drive oncogenesis in $3 \%-4 \%$ of cases. ${ }^{1}$

The team plans to start a Phase II trial this year of Bristol-Myers Squibb Co.'s Sprycel dasatinib, a marketed kinase inhibitor that blocks discoidin domain receptor tyrosine kinase 2 (DDR2). Sprycel is approved to treat acute lymphoblastic leukemia (ALL) and chronic myelogenous leukemia (CML).

The team's results also provide a rationale for testing other kinase inhibitors in lung squamous cell carcinoma (SCC).

About $70 \%$ of lung cancer patients with the SCC subtype of nonsmall cell lung cancer (NSCLC) present with advanced stage disease, said Peter Hammerman, a clinical fellow at the Dana-Farber Cancer Institute. "For these patients the treatment has changed little in the past decade. Patients typically receive a combination of two chemotherapy drugs, with about $30 \%$ of patients responding."

In contrast, treatments for the other common subtype of NSCLC, lung adenocarcinoma, have "evolved substantially over the past decade with the addition of new drugs which target specific, altered genes in the tumor, for example, EGFR [epidermal growth factor receptor] mutations and $A L K$ fusions," Hammerman said. "These targeted agents provide response rates 2-3 times better than standard chemotherapy with less toxicity."

Last December, a Cologne-based team led by Roman Thomas took a first step toward developing targeted therapies for lung SCC with the discovery that $10 \%-20 \%$ of patient tumor samples had genomic amplification of the tyrosine kinase fibroblast growth factor receptor 1 (FGFR1; CD331). 2,3

Thomas told SciBX that based on those results his team is now enrolling patients with FGFR1-amplified lung SCC in a Phase I trial of Novartis AG's BGJ398, a pan-FGFR inhibitor, in solid tumors.

Thomas is a principal investigator at the Max Planck Institute of Neurobiology and professor of internal medicine and integrated oncology at the University of Cologne.

Four months later, a group co-led by Thomas, Matthew Meyerson and Eric Haura has identified a second potential therapeutic target for lung SCC patients. The team reported that DDR2 was mutated in $3.2 \%$ of tumor samples from 277 lung SCC patients.

Meyerson is a professor in the Department of Pathology at Harvard Medical School and an associate professor of pathology and medical oncology at Dana-Farber. Haura is an associate member and director of the Lung Comprehensive Research Center at the H. Lee Moffitt Cancer Center \& Research Institute. Hammerman is a fellow in Meyerson's laboratory and first author of the study that was published in Cancer Discovery.

To determine the functional effects of the mutations, the researchers first looked at lung SCC cell lines with DDR2 mutations. Small hairpin RNA-mediated knockdown of DDR2 lowered cell proliferation compared with control shRNA-mediated knockdown of an unrelated protein.

The team next looked for kinase inhibitors that could block DDR2. Dasatinib fit the bill. The multitarget kinase inhibitor bound DDR2 with a $K_{\mathrm{d}}$ of $5.4 \mathrm{nM}$.

In a mouse xenograft model of DDR2-mutated lung SCC, dasatinib decreased tumor growth compared with vehicle.

Three other clinical-stage kinase inhibitors also bound DDR2 and reduced proliferation of DDR2-mutated lung SCC cell lines: Gleevec imatinib and Tasigna nilotinib from Novartis, and Ariad Pharmaceuticals Inc.'s ponatinib (AP24534). Gleevec is marketed for multiple hematological cancers and gastrointestinal stromal tumors (GISTs), whereas Tasigna is marketed for CML. Ponatinib is in a pivotal Phase II trial for CML and ALL and in preclinical development for solid tumors.

"These results are great news for lung SCC patients with DDR2 mutations. The study suggests that they should benefit from DDR2 inhibitors such as dasatinib and imatinib, which are FDA-approved drugs," said Chris Liang, EVP and CSO of Xcovery Inc. "This situation could be very similar to erlotinib in NSCLC patients with EGFR mutations or crizotinib in NSCLC patients with EML4-ALK fusions, where the targeted therapies have shown dramatic impacts."

Astellas Pharma Inc. and Roche's Genentech Inc. unit market Tarceva erlotinib for NSCLC and pancreatic cancer. Just this week, Pfizer's crizotinib received priority review at the FDA for ALK-positive advanced NSCLC. Xcovery has the ALK inhibitor X-396 in preclinical development for cancers with ALK alterations.

"These results are a complete and compelling preclinical story that convincingly validates DDR2 as a target," said William Shakespeare, VP of drug discovery at Ariad.

"This seems to be an unequivocal mechanism-based application for dasatinib in solid tumors," added Timothy Clackson, president of $\mathrm{R} \& \mathrm{D}$ and CSO at Ariad.

Hammerman told SciBX the investigators will be starting a Phase II trial of dasatinib in lung SCC at Dana-Farber and Massachusetts General Hospital this year. 


\section{ANALYSIS}

Bristol-Myers declined to comment.

\section{A more discerning inhibitor}

An unanswered question is whether the drug can be tolerated by patients at the doses necessary to inhibit DDR2, which is not the drug's primary target.

Indeed, dasatinib is not a particularly potent DDR2 inhibitor in cells. Based on the drug's peak serum concentration and half-life in patients, "dasatinib is unlikely to provide continuous inhibition of DDR2 even at its maximum tolerated dose," said Liang. "Clearly, more potent, and preferably more selective, DDR2 inhibitors are needed."

Shakespeare agreed. "Whether dasatinib can clinically achieve the necessary concentrations remains to be seen," he said. Other kinase inhibitors with activity against DDR2 "may turn out to be more successful clinically because of a better therapeutic window."

Although imatinib is less potent in cells, it has a better safety profile and can reach higher concentrations in humans than dasatinib and therefore could be a good option, said Liang.

Another kinase inhibitor, sorafenib, has been reported to bind DDR2 in vitro with an affinity similar to that of dasatinib. "If its cellular potency is also comparable to dasatinib, sorafenib may be able to achieve more complete and durable inhibition of DDR2" because it achieves higher concentrations in humans, Liang said.

Nexavar sorafenib is marketed by Bayer AG and Onyx Pharmaceuticals Inc. for liver and renal cancers.

Based on Ariad's studies and the data in the paper, Clackson said ponatinib appears to be "at least as potent" as dasatinib in cell-based assays. As a result, he said, lung SCC is "definitely in the mix for potential applications" of ponatinib in solid tumors.

Nathanael Gray, a coauthor on the paper, said the team is developing more selective DDR2 inhibitors as well as inhibitors that are selective for mutant DDR2 over the wild-type kinase.

Gray is an assistant professor in the Department of Biological Chemistry and Molecular Pharmacology at the Harvard Medical School and in cancer biology at Dana-Farber.
A more selective inhibitor could have decreased toxicities, but there also is the possibility that a more selective inhibitor could have reduced efficacy, said Gray. "We still don't know if we need co-inhibition of other targets in addition to DDR2 to get good effects," he noted.

The results reported in the paper are unpatented.

Kotz, J. SciBX 4(20); doi:10.1038/scibx.2011.559

Published online May 19, 2011

\section{REFERENCES}

1. Hammerman, P.S. et al. Cancer Discovery; published online April 3, 2011; doi:10.1158/2159-8274.CD-11-0005

Contact: Matthew Meyerson, Dana-Farber Cancer Institute, Boston, Mass. e-mail: Matthew_meyerson@dfci.harvard.edu

Contact: Roman K. Thomas, Max Planck Institute of Neurobiology, Cologne, Germany

e-mail: nini@nf.mpg.de

Contact: Eric B. Haura, H. Lee Moffitt Cancer Center \& Research Institute, Tampa, Fla.

e-mail: Eric.Haura@moffitt.org

2. Weiss, J. et al. Sci. Transl. Med. 2, 62 ra93 (2010)

3. Cain, C. SciBX 4(2); doi:10.1038/scibx.2011.34

\section{COMPANIES AND INSTITUTIONS MENTIONED}

Ariad Pharmaceuticals Inc. (NASDAQ:ARIA), Cambridge, Mass. Astellas Pharma Inc. (Tokyo:4503), Tokyo, Japan

Bayer AG (Xetra:BAY), Leverkusen, Germany

Bristol-Myers Squibb Co. (NYSE:BMY), New York, N.Y.

Dana-Farber Cancer Institute, Boston, Mass.

Food and Drug Administration, Silver Spring, Md.

Genentech Inc., South San Francisco, Calif.

H. Lee Moffitt Cancer Center \& Research Institute, Tampa, Fla.

Harvard Medical School, Boston, Mass.

Massachusetts General Hospital, Boston, Mass.

Max Planck Institute of Neurobiology, Cologne, Germany

Novartis AG (NYSE:NVS; SIX:NOVN), Basel, Switzerland

Onyx Pharmaceuticals Inc. (NASDAQ:ONXX), Emeryville, Calif.

Pfizer Inc. (NYSE:PFE), New York, N.Y.

Roche (SIX:ROG; OTCQX:RHHBY), Basel, Switzerland

University of Cologne, Cologne, Germany

Xcovery Inc., West Palm Beach, Fla. 